
\title{
$\begin{array}{ll}\text { Research Square } & \begin{array}{l}\text { Preprints are preliminary reports that have not undergone peer review. } \\ \text { They should not be considered conclusive, used to inform clinical practice, } \\ \text { or referenced by the media as validated information. }\end{array}\end{array}$
}

\section{Using Intervention Mapping to Develop 'ROSE': An Intervention to Support Peer Workers in Overdose Response Settings}

\section{Zahra Mamdani}

BC Centre for Disease Control https://orcid.org/0000-0002-3158-9618

Sophie McKenzie

BC Centre for Disease Control

Fred Cameron

SOLID Outreach Society

Mike Knott

SOLID Outreach Society

Jennifer Conway-Brown

RainCity Housing

Tracy Scott

RainCity Housing

Jane A. Buxton

BC Centre for Disease Control

Bernie Pauly ( $\sim$ bpauly@uvic.ca )

University of Victoria https://orcid.org/0000-0002-4845-1383

\section{Research}

Keywords: peer workers, overdose response, intervention mapping, organization, stigma, lived/living experience, substance use

Posted Date: April 13th, 2021

DOI: https://doi.org/10.21203/rs.3.rs-406184/v1

License: (9) (7) This work is licensed under a Creative Commons Attribution 4.0 International License. Read Full License 


\section{Abstract}

Background: Peer workers (those with lived/living experience of substance use working in overdose response settings) are at the forefront of overdose response initiatives in British Columbia (BC). Working in these settings can be stressful, with lasting social, mental and emotional impacts. Peer workers have also been disproportionately burdened by the current dual public health crises characterized by the onset of the COVID-19 pandemic and rise in illicit drug overdose deaths. It is therefore critical to develop supports tailored specifically to their realities.

Methods: We used the steps outlined in the Intervention Mapping (IM) framework to identify needs of peer workers and design an intervention model to support peer workers in overdose response settings. Eight peer-led focus groups were conducted at the pilot sites and other community settings to identify peer workers' needs and transcripts were analyzed using interpretive description. The strategies within the intervention model were informed by the organizational development theory as well as by the lived/living experience of peer workers.

Results: The support needs identified by peer workers were categorized into three key themes and these formed the basis of an intervention model titled 'ROSE'; R stands for Recognition of Peer Work, O for Organizational Support, S for Skill Development and E for Everyone. The ROSE model aims to facilitate culture change within organizations, leading towards a more equitable and just workplace for peer workers. This, in turn, has the potential for positive socioecological impact.

Conclusions: Centering lived/living experience in the Intervention Mapping process led us to develop a framework for supporting peer workers in BC. The ROSE model can be used as a baseline for other organizations employing peer workers.

\section{Contributions To The Literature}

- The Intervention Mapping (IM) framework is applied to the creation of an intervention model for supporting peer workers in overdose response settings. Our research demonstrates that IM is an effective tool for organizational planning and community engagement.

- Combining health promotion theories with experiential knowledge facilitates the creation of robust interventions to support peer workers.

- Our findings on how to best support peer workers in BC are the first of their kind laying a firm, practical foundation for organizations employing people with lived/living experience of substance use.

\section{Background}

On April 14th, 2016 the British Columbia (BC) Provincial Health Officer declared a public health emergency in response to a dramatic rise in drug overdoses (1). Peer workers ${ }^{1}$ are at the forefront of overdose response in $\mathrm{BC}$ and critical in the province's response to a devastating public health crisis (2-4). Peer workers perform a variety of roles, including responding to overdoses, distribution of naloxone and other harm reduction supplies, peer witnessing of drug use, referrals to services such as housing agencies, advocacy, outreach, and research (5). Employing peers in overdose response settings is critical in increasing the accessibility and acceptability of programs for people who use substances (6-9). There is an increasing body of knowledge that supports the impact of peer work on reducing harms associated with drug use and structural violence (8-12). The onset of the Coronavirus Disease of 2019 (COVID-19) pandemic has been accompanied by escalating rates of overdoses and increased work and stress for peer workers due to implementation of public health measures resulting in closures and reduced hours of organizations providing services to people who use drugs (PWUD) $(13,14)$.

Although peer work has multiple benefits for service users and employees alike, the work is stressful, with lasting emotional and mental health effects ( 9,12 , 15-18). Despite working in stressful environments, unlike other healthcare providers and first responders $(19,20)$, peer workers usually lack access to occupational supports available to employees. This workplace discrimination is situated more broadly within a legal framework that criminalizes drug use (21, 22). As such, there are few resources or programs for people with lived/living experience of substance use who work in overdose response settings (23-26). This lack of organizational supports for peer workers can lead to compassion fatigue and burnout (27-29). There is a critical need for supports for peer workers that recognize their unique positioning as people with lived/living experience within a professional setting as well as the often-adverse effects of working in overdose response environments.

Intervention Mapping (IM) creates theory- and evidence-based health promotion programs via community-based development processes to ensure that the intervention adequately addresses community needs $(30,31)$. As such, we identified IM as an appropriate framework to use within the Peer-to-Peer Project, which aims to identify, implement and evaluate peer-led interventions that are feasible and effective for supporting peer workers in overdose response settings in $\mathrm{BC}$ (32). IM is informed by socio-ecological theory with a prioritizing of multi-level intervention planning by evaluating individual, inter-personal, organizational, community and societal influences on health outcomes $(30,31)$. In this paper, we describe in detail how a support intervention for peer workers, titled 'ROSE', was conceptualized, with recommendations for other organizations employing peer workers to tailor the intervention components for their respective settings.

${ }^{1}$ Those with past or present substance use experience who use that lived experience to inform their professional work $(8,9,63)$.

\section{Methods}

The ROSE model (Fig. 1, described later) was developed through a community-based research project conducted in collaboration with two organizations in BC: 1) SOLID Outreach Society - a peer-led organization on Vancouver Island that educates, advocates and provides services for individuals that use substances (33), and 2) RainCity Housing - a not-for-profit, housing-first organization in Vancouver Coastal and Fraser regions that provides housing and support services for people living with mental health, substance use, and other challenges (34). The methods described here are based on the COREQ checklist (attached)(35) . 
The project team consists of academic researchers and representatives from each organization, including peer research assistants (PRAs). The PRAs were people with lived/living experience of substance use who were selected by the respective partnering organizations to be part of the project team and represent the voices of other peer workers within their organizations. As such, all PRAs had robust and trusted relationships with individuals at each organization. Further, all PRAs were trained in qualitative research practices and ethics. The team consists of a diversity of ages, genders, and educational backgrounds.

\section{Designing the model}

The Intervention Mapping (IM) protocol developed by Bartholomew et al. in 1998 follows a structured approach guided by a six-step protocol which describes the iterative path from problem identification to evaluation of the intervention (31). Each of the six steps of IM comprises several tasks which integrate theory and evidence (31).

To design the ROSE model, we followed the steps as outlined by IM. These steps include: needs assessment, specification of program objectives, selection of theory-based methods and strategies for addressing identified needs, creating a program plan, creating an implementation plan and creating an evaluation plan $(30,31)$. Below we describe application of the steps of IM to design the ROSE model.

\section{Step 1: Needs Assessment}

Eight focus groups with 31 participants in total were conducted between November 2018 and March 2019; two in the Fraser region, two in Vancouver and four in Victoria. Details on recruitment of participants, interview processes, and data analysis are described in two previously-published articles (36, 37).

Focus groups were facilitated by PRAs at RainCity Housing and SOLID Outreach. An additional focus group was conducted at an emergency shelter with an overdose prevention site in Victoria, to encourage a diversity in peer worker perspectives. An academic researcher was also present to take field notes. All focus group participants gave consent and were given the option to complete a demographic questionnaire. The consent form included a description of the project goals, the biography of the key researchers and their contact information. A diversity of demographic characteristics was represented within the participant group. Just over half of the participants were male (55\%). Of those that completed the questions related to age and education $(n=18,58 \%$ of total), $56 \%$ were over the age of 40 and all reported having received at least some high school education. The demographic profile of these participants is presented in Table 1.

Table 1

Demographic Characteristics of Focus Group participants

\begin{tabular}{|ll|}
\hline Gender & $\%$ out of $\mathbf{N}=\mathbf{3 1}$ \\
Male & $\mathbf{5 5 \%}$ \\
\hline Age & $\mathbf{4 5 \%}$ \\
Under 20 & $\%$ out of $\mathbf{N}=\mathbf{3 1}$ \\
$21-30$ & $0 \%$ \\
$31-40$ & $6 \%$ \\
$41-50$ & $19 \%$ \\
$51-60$ & $13 \%$ \\
$61-70$ & $6 \%$ \\
$71+$ & $10 \%$ \\
Unknown & $3 \%$ \\
\hline Highest Level of Education & $42 \%$ \\
Some high school & $\%$ out of $\mathbf{N}=\mathbf{3 1}$ \\
Completed high school & $23 \%$ \\
Some community college or technical school & $6 \%$ \\
Completed comm. college or technical school & $6 \%$ \\
Some university & $3 \%$ \\
Completed Bachelor's degree & $6 \%$ \\
Post Graduate Training & $3 \%$ \\
Unknown & $42 \%$ \\
\hline & \\
\hline
\end{tabular}

Using thematic analysis to comprehensively identify, analyze, organize, and report themes $(38,39)$, a list of issues faced by peer workers was compiled as a first step in identifying their support needs. The coding framework was initially developed by the academic researchers then shared with PRAs for their input 
and validation. The final coding transcript was inputted into NVivo (QSR International, version 12) where one academic researcher coded the data iteratively and reflexively, with regular check-in meetings with the rest of the team, including the PRAs for interpretive description of the meaning behind the quotes.

\section{Step 2: Specification of Program Objectives}

Using a participatory coding process, described in previously-published articles the project team sorted each of the support needs into overarching themes $(36,37)$. Next, using interpretive description to delve deeper into the quotes and situate the themes within a real-world context $(40)$, the project team categorized the issues according to levels of the Socio-Ecological Model (SEM), "a framework for understanding the multiple levels of a social system and interactions between individuals and environment within this system" (41). The master list of issues faced by peer workers sorted into different SEM levels is presented in Table 2 below. Once sorted into themes and SEM levels, the identified needs allowed the team to determine the program objectives.

Table 2

Master list of issues identified by peer workers from a socio-ecological perspective

\begin{tabular}{|c|c|}
\hline $\begin{array}{l}\text { Socio-Ecological } \\
\text { Model Level }\end{array}$ & Issues faced by peer workers \\
\hline \multirow[t]{2}{*}{ Individual } & $\begin{array}{l}\text { - Lack of adequate skills and confidence. Examples of topics identified include recognizing signs and symptoms of mental health } \\
\text { disorders and challenges, self-defence, conflict resolution, first aid, and communication. }\end{array}$ \\
\hline & - Personal health and lack of opportunities for self-care and de-stressing. \\
\hline \multirow[t]{2}{*}{ Inter-personal } & Lack of access to peer-to-peer debriefing. \\
\hline & Strained relationships with other professionals e.g. Police and paramedics. \\
\hline \multirow[t]{4}{*}{ Organizational } & - Inequity in the workplace including inequitable pay, differential treatment of peer workers. \\
\hline & Precarious/ unstable jobs. \\
\hline & Lack of respect and recognition at work. \\
\hline & - Lack of role clarity. \\
\hline \multirow[t]{2}{*}{ Community } & - Lack of respect and recognition for peer workers in the community (by other professionals). \\
\hline & - Lack of access to and awareness of community resources such as government issued ID cards, detox, etc. \\
\hline \multirow[t]{4}{*}{ Policy or Society } & Stigma. \\
\hline & - Low minimum wage. \\
\hline & · Harmful policies such as criminalization of drug use. \\
\hline & - Lack of adequate housing supports. \\
\hline
\end{tabular}

\section{Step 3: Selection of theory-based methods and strategies for addressing identified needs}

Our findings indicated a need for support interventions spanning various levels of the SEM, however given our project's goal to improve supports for peer workers in overdose response settings, we identified organizational interventions as a priority. We specifically considered the Ottawa Principles of Health Promotion (42), which include enabling equity by providing a supportive environment and access to information.

Given our focus on organizational-level interventions, the Organizational Development Theory was chosen as the primary theory for intervention planning. This theory is defined as "a system-wide process of applying behavioural science knowledge to the planned change and development of the strategies, design components, and processes that enable organizations to be effective" (43). Specifically, we focused on creating an intervention that would affect the organizational culture, i.e. assumptions and beliefs which govern the behaviour of members of the organization, and organizational climate, i.e. the personality of the organization (43).

\section{Step 4: Creating a Program Plan}

Based on the first three steps of IM, the PRAs identified three overarching themes which they considered to be the most prominent needs. These include recognition of peer work, organizational support, and skill development. These three themes formed the basis of the intervention model, from which the team collaboratively came up with a list of potential model names. Eventually, through an anonymous voting process, the team named the intervention model 'ROSE', with each letter standing for one of the three themes (recognition, organizational support, skill development). The E in the ROSE model emphasizes that the resources developed are for Everyone, and highlights its inclusivity and the commitment of the peer workers at the pilot sites to make the resources available to all organizations across BC (see Fig. 1).

Once the intervention was named, strategies to tackle issues within each component of the intervention were decided upon during the bi-weekly meetings with the project team. This step was informed by evidence from literature and by peer worker suggestions. This led to the creation of a comprehensive yet feasible intervention model to address the identified needs of peer workers in overdose response settings.

Step 5: Creating an Implementation Plan

Page $4 / 12$ 
Next, the project team defined the scope of the intervention and decided on the project activities for each pilot site. Furthermore, the sequence of activities was determined, prioritizing the strategies that were easiest to implement and had potentially high impact. PRAs and representatives from each partner site decided on the start and end dates for each activity, milestones, budget, and task assignment, which was documented as detailed site-specific action plans.

To guide the implementation of the interventions, the Normalization Process Theory (NPT) was utilized. NPT is a mid-range implementation theory that explores how components of an intervention are implemented, routinely embedded in everyday practice, and integrated within organizational settings (44). Consistent with NPT, we considered four theoretical constructs: (1) coherence (how people understand the practice) (2) cognitive participation (how people engage in a practice); (3) collective action (how the practice interacts with existing practices) and; (4) reflexive monitoring (how a practice is understood) (44). NPT is interested in both the implementation process as well as the outcomes of the intervention (44).

NPT has been applied to numerous health studies and helps to guide and explain implementation of new processes (45). As such, it was an appropriate theory to inform the ROSE model.

\section{Step 6: Creating an Evaluation Plan}

The final step of intervention mapping was to create the evaluation plan. Consistent with NPT, we developed both a process and outcome evaluation plan.

To assess the process of implementing several aspects of the ROSE model, we conducted qualitative interviews with both implementors and recipients of the intervention, i.e., the organizational managers and peer workers. This evaluation was informed by NPT and conducted in collaboration with the partner organizations.

To assess the outcomes of the interventions, a survey informed by the focus group findings was conducted. This survey consisted of demographic questions, measures of peer workers' perceptions of health and quality of life, substance use patterns and working conditions. The majority of the survey questions were adapted from validated tools with good psychometric properties, including the Canadian Community Health Survey (46), Short Form - 12 (SF-12) Health survey (47), the Professional Quality of Life Scale (ProQOL) (48), and the Job Satisfaction Survey (49). The survey was conducted both prior to implementation of the interventions as well as one year after implementation in order to assess the change in these measures attributed to the interventions.

In this paper we report on the development of the ROSE model. The implementation process and the results of the evaluation will be reported in a subsequent paper.

\section{Results \\ The Intervention}

The ROSE model stands for R: Recognition of Peer Work, O: Organizational Support, S: Skill Development and E: for Everyone (Fig. 1). The overarching aim of the ROSE model is to increase support for peer workers in overdose response settings. The objectives of the ROSE model are to: 1) Facilitate equitable access to resources for peer workers, enabling them to stay motivated and work optimally in a stressful work setting, with reduced emotional, mental and social stress, 2) Provide training and education for peer workers to improve their skills and gain professional self-confidence, and 3) Increase awareness and recognition among individuals without lived/living experience about the crucial work done by peer workers in overdose response settings.

Through these objectives, the ROSE model aims to facilitate culture change within organizations, leading towards a more equitable and just workplace. This, in turn, will lead to a positive impact at various socio-ecological levels, including improved self-confidence of peer workers at the individual level, formation of social networks and relationships with colleagues and other professionals at the interpersonal and community levels, more equitable and just workplaces at the organizational level, and ultimately a more accepting and less stigmatizing society.

Each component of the ROSE model consisted of several strategies informed by theory, evidence, and the lived/living experience of PRAs (Fig. 1). Details of the strategies within each component are specified below.

\section{Recognition of Peer Work}

As described in another paper (37), one of the top issues encountered by peer workers is that they felt they are not taken seriously or given due respect by their work colleagues or other professionals they encounter in their work. This is manifested in various forms, including inequity in the workplace, lack of basic workplace resources, and strained relationships with other service providers (37).

To address this issue, three primary strategies were identified and implemented under the "Recognition" component of the ROSE model. First, peer workers identified the need for basic resources such as photo identity cards (ID cards) and business cards as tangible symbols of professionalism, authority and validity within their roles. Pilot sites were provided with portable ID card printers and business card templates so that all peer workers employed could receive their individual photo IDs and business cards.

Secondly, to create awareness about the work done by peer workers, a video titled \#PeerLife, featuring a day in the life of a peer worker, was developed (50). This video featured the story of four peer workers, one from each of the pilot sites, and their day-to-day work in the face of the overdose crisis. The video highlights the harsh realities faced by peer workers and encourages recognition and appreciation for their work. This video is available on YouTube, has been promoted through social media, and featured on the Toward the Heart website. 
A third strategy that was recommended was meet and greet events between peer workers and other professionals including police and paramedics. The purpose of these events was to foster relationship-building and to raise awareness about the crucial roles fulfilled by peer workers.

The strategies under the "Recognition" component span across multiple levels of the socio-ecological model. Provision of photo IDs and business cards to peer workers, for example, constitute organizational-level interventions, while meet and greet events foster inter-personal relationships between peer workers and other professionals. These relationships, in turn, can help to improve peer workers' work experience (organizational-level) and may address negative attitudes and stigma towards peer workers (societal-level). Similarly, the \#PeerLife video, which creates awareness about the role of peer workers is a societallevel intervention since it is a first step towards addressing stigma and negative attitudes towards PWUD (see Table 2).

\section{Organizational Support}

Peer workers indicated that their work is stressful and despite their wholehearted commitment to the job $(36,37)$, their lack of organizational and mental health supports to mitigate their stresses creates notable dissatisfaction. Lack of such resources often led to low morale and burnout (37).

As highlighted in another paper, many peer workers indicated a lack of job clarity and formalized contracts with their organizations, leading to poor working conditions and relegation to menial labour by supervisors and co-workers (37). Inequitable pay, despite the similar nature of work done by support workers without lived/living experience, was another issue.

The "Organizational Support" component of the ROSE model consists of several strategies to address these issues. To create role clarity, formal job descriptions were created, which solidified the role of peer workers and suggested a living wage based on BC's peer worker pay standards (23, 51 ). Formal employment contracts which detailed the terms and expectations of employment were also developed. These documents were implemented at the pilot sites and templates of these documents were compiled into a Best Practice Manual to Support Peer Workers and made publicly available for other organizations to adapt based on their needs (52).

Additionally, two roles were created at one of the pilot sites which is a peer-led organization. The first one was the role of the Peer Supporter. A person with lived/living experience of substance use was hired into this role to provide a listening ear and peer-to-peer debriefing. This role was informed by literature which indicates that shared experience helps to facilitate trust, understanding and a special bond of care and comfort (36).

The second role was that of a Systems Navigator. A person with lived/ living experience of substance use was hired as a Systems Navigator to support peer workers in navigating access to external services. These included a variety of supports such as assisting with access to harm reduction services, accompanying peer workers to healthcare visits, providing legal support, supporting peer workers to apply for their government identification cards, providing assistance to complete housing applications and income assistance forms, providing referrals and reference letters for housing applications, and referring peer workers to detox or treatment, if desired. The Systems Navigator also builds relationships with external service providers and researching services to acquaint themselves with these systems and provide easy referrals to peer workers. As such, the Systems Navigator can assist in decolonizing access to external resources by acting as the stepping stone that is absent for people who use substances in most administrative systems.

Another strategy under the "Organizational Support" component included teambuilding days. These were organized to boost the morale of peer workers and allow them to de-stress and unwind. For each pilot site, teambuilding days were organized twice a year and included fun and celebratory activities, such as bowling and holiday parties.

In addition to addressing the needs of the peer workers identified during the focus groups, the "Organizational Support" component of the intervention also provided resources that were identified during the bi-weekly check-in and progress meetings with the whole team, including the PRAs. One such resource was the need for pulse oximeters in response to increasing reports in BC of substances containing mixtures of opioids and benzodiazepines and the identification of unregulated etizolam in urine drug screens (53-55), causing people to remain unconscious long after blood oxygen levels have returned to the normal range after naloxone administration (53-55). Pulse oximeters aid in identifying when oxygen levels are within normal range and rescue breaths are not needed, which is of particular importance since the onset of the COVID-19 pandemic.

Like strategies in the "Recognition" component, the "Organizational Support" interventions span across multiple levels of the socio-ecological model. For example, the Peer Supporter role is an organizational-level intervention, however, peer workers may realize improved mental health through engagement with the role, which is an individual-level factor. Similarly, the hiring of a Systems Navigator is done at the organizational level, but through relationship-building and referrals, this role is able to increase peer workers' access to external community resources, thus also operating at the community level. Teambuilding days help to improve relationships between colleagues (interpersonal) and boost morale and motivation (individual). Through the implementation of organizational supports, the ROSE model has the potential to challenge the norms and address the negative attitudes and stigma towards peer workers both within the organization (organizational) and in society in general (societal) (See Table 2).

\section{Skill Development}

During the focus groups, peer workers identified skill development as a need and suggested the topics they would find helpful in their work. As described in another paper, peer workers felt that organizations tend to value formal education and certification over their lived/living experience (37). As such, peer workers were keen to gain some formal training and certifications to increase their self-confidence and capacity. Identified training needs ranged from technical skills to people skills and self-care skills. Technical skills identified included first aid and CPR, recognition of signs and symptoms of mental health disorders, naloxone administration and use of pulse oximeters. Under people skills, peer workers identified the need for training in conflict resolution and deescalation, communication skills, peer debriefing skills and cultural safety. Self-care skills included mindfulness and self-defence. 
For some topics, such as first aid, well-recognized external training already existed. In such cases, peer workers were supported to attend these external trainings and earn certification. In addition to providing peer worker training on topics identified during the focus groups, several other training materials were developed to meet the circumstantial needs of peer workers. For example, with the onset of COVID-19, information sheets and training videos on responding to overdoses in light of COVID-19 were developed.

For topics which lacked existing training tailored for peer workers, the team is currently developing a standardized BC peer worker training curriculum. It is critical that peer worker training or capacity-building be tailored to the realities of people who use substances as many workplace training programs are designed for people without lived/living experience of criminalization or other impacts of drug use.

\section{Theoretical underpinnings}

The strategies within each component of the ROSE model are strongly aligned with the organizational development theory as the ROSE model aspires to positively change the organizational culture and climate. For example, by providing equitable access to supports for peer workers within their organizations, the ROSE model aspires to challenge the norm and take the first step towards changing the otherwise discriminatory and negative attitudes towards peer workers. The ROSE model also has the potential to facilitate relationship-building between peer workers and other professionals, instilling positive attitudes and behaviours that may translate into a way of life over time $(56,57)$. In this way, the ROSE model can have organization- and community- wide ripple effects, as it will potentially allow all the staff at the organization to value an inclusive and just workplace, changing the organizational culture and climate. The implementation of the ROSE model is also aligned with NPT because it is aimed at not only instilling a temporary change in behaviour and attitudes within an organization, but hopes to achieve long-term effects which become routinely embedded in everyday practice and integrated within organizational settings (44).

\section{Discussion}

As highlighted in our previous paper (37), peer workers face multiple sources of stress both personally and work. These stressors are a reflection of the socioeconomic marginalization that PWUD face in employment contexts, which is rooted in systemic stigma (24-26). The issues faced by peer workers span across multiple levels of the socio-ecological model, calling for multi-levelled interventions. The ROSE model is an organization-level intervention that can potentially have impacts spanning across multiple levels.

A review of workplace interventions indicates that the majority of workplace interventions aim to reduce work-related stress and take one of three forms: primary, which are typically for all employees and have a focus on prevention, secondary which target employees who have been exposed to risk factors and provide employees with knowledge and skills to cope with the stressor, and tertiary which focus on employees who are experiencing distress and need assistance in recovering from stress-related symptoms (58). Interventions in these three forms are limited in that they tend to focus on individuals rather than on organizations. The ROSE model does contain a mixture of primary, secondary and tertiary strategies, but goes beyond the individual; the strategies within the ROSE model focus on organizational culture change. In doing so, the changes within organizations are sustainable as they become routinely embedded in everyday practice within the organizations.

In designing the ROSE model, the Peer-to-Peer team also considered the success of similar interventions in different contexts. For example, past studies have indicated that emotionally-supportive exchanges between peers can foster feelings of being accepted, cared for, empathized, respected, and valued despite profound personal difficulties (59-61). This evidence supported the creation of the role of a Peer Supporter within the "Organizational Support" component of the intervention. Furthermore, according to a review of interventions for reducing stigma, educational interventions can help to improve attitudes of individuals without lived/living experience towards PWUD (62). As such, many of the interventions in the "Recognition" component of the ROSE model are centred on the idea of creating awareness of the work peer workers do in order to address the negative attitudes towards them. The review also indicated that interventions which create direct contact with PWUD and focus on relationship-building with people with lived/living experience of substance use can address social and structural stigma. This informed the implementation of meet and greet events between peer workers and service providers they often encounter, such as police officers.

The authors recognize that even though the interventions within the ROSE model are informed by theory and evidence, their ability to impact societal level factors such as stigma is minimal. This stigma is deeply embedded in the long history of drug prohibition in Canada which continues to criminalize substance use $(21,22)$, leading to inequity in income, education and housing for PWUD. Unless measures to address these underlying issues are taken, such as decriminalization, peer workers may never fully feel supported. Yet, while organization-based interventions may be impeded by the continued criminalization of drug use, the ROSE model is an organizational intervention with potential ripples and impacts at multiple levels in the socio-ecological context.

The ROSE model has many strengths. Firstly, the participants during the focus groups (needs assessment) had a diversity of age, gender, roles, geography, and education level. This provided a range of views on the support needs of peer workers and potential solutions to meet the needs. Second, each of the strategies that were decided upon were strongly informed by theory and evidence from literature, which increases the chances of success. In addition, and most importantly, the intervention was shaped by the lived/living experience of PWUD and centred the voices and opinions of peer workers. This allowed peer workers to have their say over decisions that affect their lives and the lives of their communities. Furthermore, the engagement of peer workers ensures that the strategies implemented are not only relevant but also acceptable for peer workers at the partner sites, and beyond. By keeping peer workers at the centre of the intervention planning and implementation, the ROSE model challenges the culture of oppression rooted in the colonial endeavour to make decisions for marginalized populations rather than with them.

Despite these strengths, the ROSE model has some limitations. One limitation is that the intervention is based on needs assessment data from focus groups in which participants may have been hesitant to express their opinions due to fear of judgement from other participants or of jeopardizing their jobs. This 
concern was mitigated by keeping the focus groups small, and by ensuring that no managers were present. Furthermore, the focus groups were facilitated by PRAs to promote a power balance and to ensure that participants feel comfortable to provide their honest opinions. Another limitation of the intervention is that the partner sites were located within four metropolitan or large urban centers in BC and the experiences and needs of peer workers in other settings, especially in rural settings, may be different. The partner sites, however, do represent diversity in type i.e., housing agency versus non-housing, and geography i.e., four different cities. More research is warranted to test the applicability of the intervention for peer workers in other contexts in BC and in Canada.

\section{Conclusion}

Using the IM process, we developed the ROSE model which consists of three components (Recognition of Peer Work, Organizational Support and Skill Development for Everyone). Each component includes a set of strategies designed to meet the specific objectives of the intervention. The development of the ROSE model was rooted in the lived and living experience of peer workers and was informed by previous literature and health promotion theories. Importantly, the ROSE model is an organizational level intervention which has the potential for impact at individual, inter-personal, organizational, community and systems levels and requires further evaluation to determine efficacy of the intervention.

\section{Abbreviations}

BC - British Columbia,

BCCDC - British Columbia Centre for Disease Control

COVID-19 - Coronavirus Disease of 2019

IM - intervention mapping

PRA - peer research assistant

PWUD - people who use drugs

ROSE - Recognition of peer work, Organizational support and Skill Development for Everyone

\section{Declarations}

\section{Ethics approval and consent to participate:}

The study received Research Ethics approval from the University of British Columbia Research Ethics Board (REB \#: H18-00867). All focus group participants provided written consent for participation.

\section{Consent for publication:}

Not applicable.

\section{Availability of data and materials:}

The datasets used and/or analyzed during the current study are not publicly available due to the small nature of peer networks and potential for identification, but are available from the corresponding author on reasonable request.

\section{Competing interests:}

The authors have no actual or potential conflict of interest to declare.

\section{Funding:}

This work was supported by Health Canada's Substance Use and Addictions Program (Grant Arrangement Number: 1718-HQ-000030). The funders had no role in the study design, data collection, analysis and interpretation of data or in the writing of the manuscript.

\section{Authors' contributions:}

ZM, BP and JB conceptualized the study and formulated the methodological framework. ZM, BP, JB, JCB, FC and MK were involved in data collection. ZM completed formal data analysis, conducted the investigation process, and administrated the project. FC, TS, MK, and JCB validated the data. ZM and SM wrote the initial draft of the article. ZM, BP, JB, SM, FC, TS, JCB, and MK reviewed and edited the manuscript. All authors read and approved the final manuscript.

\section{Acknowledgements:}

The authors would like to thank the past and present members of the Peer-to-Peer Research Team and the pilot organizations for their tireless efforts in implementing the project. We would also like to acknowledge the Harm Reduction Services team at the British Columbia Centre for Disease Control (BCCDC) 
for their support with this project. We are indebted to the peer workers across the province who are dedicated to being in the frontline to save lives from drug overdoses during the ongoing dual public health emergencies in BC.

\section{References}

1. BC Gov News. Provincial health officer declares public health emergency | BC Gov News [Internet]. BC Gov News. 2016 [cited 2019 Sep 19]. Available from: https://news.gov.bc.ca/releases/2016HLTH0026-000568

2. Smart A. Peer Support workers on front lines of the OD crisis need support too: Experts [Internet]. National Post; 2018. Available from: https://nationalpost.com/pmn/news-pmn/canada-news-pmn/peer-support-workers-on-front-lines-of-the-od-crisis-need-support-too-experts

3. Law M. Peer support workers in Quesnel work to reduce overdose deaths in the north. Quesnel Cariboo Observer [Internet]. 2018; Available from: https://www.quesnelobserver.com/news/peer-support-workers-in-quesnel-work-to-reduce-overdose-deaths-in-the-north/

4. Zeidler M. "People have to be bold" | CBC News. 2019.

5. Marshall Z, Dechman MK, Minichiello A, Alcock L, Harris GE. Peering into the literature: A systematic review of the roles of people who inject drugs in harm reduction initiatives. Vol. 151, Drug and Alcohol Dependence. Elsevier Ireland Ltd; 2015. p. 1-14.

6. Greer A, Luchenski S, Amlani A, Lacroix K, Burmeister C, Buxton J. Peer engagement in harm reduction strategies and services: A critical case study and evaluation framework from British Columbia, Canada. BMC Public Health [Internet]. 2016 Dec 27 [cited 2020 Jan 3];16(1):1. Available from: https://doi.org/10.1186/s12889-016-3136-4

7. Pauly B, Wallace B, Pagan F, Phillips J, Wilson M, Hobbs H, et al. Impact of overdose prevention sites during a public health emergency in Victoria, Canada. Panagiotoglou D, editor. PLoS One [Internet]. 2020 May 21 [cited 2020 Jul 9];15(5):e0229208. Available from: https://dx.plos.org/10.1371/journal.pone.0229208

8. Bardwell G, Kerr T, Boyd J, McNeil R. Chracterizing peer roles in an overdose crisis: Preferences for peer workers in overdose response programs in emergency shelters. Drug Alcohol Depend [Internet]. 2018 Sep 1 [cited 2020 Apr 21];190:6-8. Available from: http://www.ncbi.nlm.nih.gov/pubmed/29960202

9. Kennedy MC, Boyd J, Mayer S, Collins A, Kerr T, McNeil R. Peer worker involvement in low-threshold supervised consumption facilities in the context of an overdose epidemic in Vancouver, Canada. Soc Sci Med. 2019 Mar 1;225:60-8.

10. Gillespie A, Lasu B, Sawatzky A. Peer Support Models for Harm Reduction Services: A Literature Review for The Wellington Guelph Drug Strategy (WGDS) [Internet]. 2018. p. 1-61. Available from: https://atrium.lib.uoguelph.ca/xmlui/handle/10214/8902

11. Greer A. Work Experiences and Conditions Among People Who use Drugs Engaged in Peer Work: A Critical Examination of Peer Work in British Columbia, Canada [Internet]. 2019 [cited 2020 Apr 21]. Available from: https://open.library.ubc.ca/clRcle/collections/ubctheses/24/items/1.0383396

12. Wagner KD, Davidson PJ, Iverson E, Washburn R, Burke E, Kral AH, et al. "I felt like a superhero": The experience of responding to drug overdose among individuals trained in overdose prevention. Int J Drug Policy [Internet]. 2013;25(1):1. Available from: https://doi.org/10.1016/j.drugpo.2013.07.003

13. Pagliaro J. City's busiest supervised injection site to reopen after month-long closure over COVID-19. The Star [Internet]. 2020 [cited 2020 Jun 4]; Available from: https://www.thestar.com/news/city_hall/2020/04/16/citys-busiest-supervised-injection-site-to-reopen-after-month-long-closure-over-covid-19.html

14. Woo A. Virus measures may be hurting overdose prevention in Vancouver, official says. The Globe and Mail [Internet]. 2020 [cited 2020 Jun 4]; Available from: https://www.theglobeandmail.com/canada/british-columbia/article-virus-measures-may-be-hurting-overdose-prevention-in-vancouver/

15. Bardwell G, Anderson S, Richardson L, Bird L, Lampkin H, Small W, et al. The perspectives of structurally vulnerable people who use drugs on volunteer stipends and work experiences provided through a drug user organization: Opportunities and limitations. Int J Drug Policy [Internet]. 2018 May 1 [cited 2020 Apr 21];55:40-6. Available from: http://www.ncbi.nlm.nih.gov/pubmed/29501928

16. Shepard BC. Between harm reduction, loss and wellness: on the occupational hazards of work. Harm Reduct J [Internet]. 2013 [cited 2020 Jan 3];10(1):5. Available from: http://harmreductionjournal.biomedcentral.com/articles/10.1186/1477-7517-10-5

17. Declaire C. On the front line of a crisis, overdose-prevention staff could use some help. CBC News [Internet]. 2018 [cited 2020 Jan 3]; Available from: https://www.cbc.ca/news/health/overdose-prevention-sites-mental-health-frontline-workers-1.4893867

18. Teti M, Bowleg L, Spencer S. Who Helps the Helpers? A Clinical Supervision Strategy to Support Peers and Health Educators Who Deliver Sexual Risk Reduction Interventions to Women Living With HIV/AIDS. J HIV AIDS Soc Serv. 2009;8(4).

19. Johnson L. "We're starving for it": Paramedics get new resiliency training due to overdose crisis | CBC News. 2017 [cited 2020 Jan 3]; Available from: https://www.cbc.ca/news/canada/british-columbia/first-responders-overdose-trauma-stress-1.4115497

20. Joint Task Force on Overdose Prevention and Response. Progress Update on B.C.'s Response to the Opioid Overdose Public Health Emergency [Internet] 2017 [cited 2020 Jan 3]. Available from: https://www2.gov.bc.ca/assets/gov/health/about-bc-s-health-care-system/office-of-the-provincial-healthofficer/overdose-response-progress-update-march2017.pdf

21. Boyd SC. More Harm Than Good: Drug Policy in Canada [Internet]. Winnipeg, Manitoba: Fernwood Publishing; 2017 [cited 2020 Jul 9 ]. Available from: https://www.chapters.indigo.ca/en-ca/books/more-harm-than-good-drug/9781552668627-item.html

22. Boyd N. High Society: Legal and Illegal Drugs in Canada [Internet]. Toronto, Ontario: Key Porter Books; 1991 [cited 2020 Jul 9]. Available from: https://books.google.ca/books/about/High_Society.html?id=UqxIAAAAYAAJ\&redir_esc=y

23. Greer A, Buxton J. A guide for paying peer research assistants: Challenges and opportunities (version 2). [Internet]. BC Centre for Disease Control; 2018. Available from: http://www.bccdc.ca/resource-gallery/Documents/Educational Materials/Epid/Other/peer_PEEP_payment_2018.pdf 
24. Griffiths CA, Hancock-Johnson E. The experiences of paid formal lived experience workers within a secure mental health service. J Ment Heal Training, Educ Pract. 2016;12(5).

25. Roose RJ, Cockerham-Colas L, Soloway I, Batchelder A, Litwin AH. Reducing Barriers to Hepatitis C Treatment among Drug Users: An Integrated Hepatitis C Peer Education and Support Program. J Health Care Poor Underserved. 2014;25(2).

26. Salmon A, Browne AJ, Pederson A. 'Now we call it research': Participatory health research involving marginalized women who use drugs. Nurs Inq [Internet]. 2010;17(4). Available from: https://doi.org/10.1111/j.1440-1800.2010.00507.x

27. Figley C. Treating Compassion Fatigue [Internet]. Brunner - Routledge. New York; 2002 [cited 2020 May 1]. Available from: https://books.google.ca/books? $\mathrm{hl}=$ en\&lr=\&id=2qyVRQ8y7SkC\&oi=fnd\&pg=PP1\&dq=compassion+fatigue+mental+health\&ots=XLMGiPtXQL\&sig=e7MH7q4AWfUYVe9KZsYq0SyxzmQ\#v= fatigue mental health\&f=false

28. Sheppard K. Compassion fatigue among registered nurses: Connecting theory and research. Appl Nurs Res. 2015 Feb 1;28(1):57-9.

29. Yoder EA. Compassion fatigue in nurses. Appl Nurs Res. 2010 Nov 1;23(4):191-7.

30. Fernandez ME, Ruiter RAC, Markham CM, Kok G. Intervention mapping: Theory-and evidence-based health promotion program planning: Perspective and examples. Front Public Heal. 2019;7(AUG).

31. Bartholomew LK, Parcel GS, Kok G. Intervention Mapping: A Process for Developing Theory- and Evidence-Based Health Education Programs [Internet]. Vol. 25, Health Education and Behavior. SAGE Publications Inc.; 1998 [cited 2021 Jan 6]. p. 545-63. Available from:

https://pubmed.ncbi.nlm.nih.gov/9768376/

32. Peer-2-Peer Project. The ROSE Initiative [Internet]. 2019 [cited 2020 Jun 18]. Available from: https://towardtheheart.com/assets/uploads/1576697632Pzvl9acqgoqPJDiDEcVRqBnQckNXaE5LdSXe2Kv.pdf

33. Solid Outreach. Solid Outreach [Internet]. 2020 [cited 2020 Jul 21]. Available from: https://solidvictoria.org/

34. RainCity Housing. RainCity Housing [Internet]. 2020 [cited 2020 Jul 21]. Available from: https://www.raincityhousing.org/\#

35. Tong A, Sainsbury P, Craig J. Consolidated criteria for reporting qualitative research (COREQ): a 32 -item checklist for interviews and focus groups. Int $J$ Qual Heal Care [Internet]. 2007 Sep 16 [cited 2021 Apr 5];19(6):349-57. Available from: https://academic.oup.com/intqhc/articlelookup/doi/10.1093/intqhc/mzm042

36. Pauly B (Bernie), Mamdani Z, Mesley L, McKenzie S, Cameron F, Edwards D, et al. "It's an emotional roller coaster... But sometimes it's fucking awesome": Meaning and motivation of work for peers in overdose response environments in British Columbia. Int J Drug Policy [Internet]. 2020 Feb 1 [cited 2021 Feb 22];88:103015. Available from: https://linkinghub.elsevier.com/retrieve/pii/S0955395920303534

37. Mamdani Z, Mckenzie S, Pauly B (Bernie), Cameron F, Conway-Brown J, Edwards D, et al. "Running myself ragged”: Stressors Faced by Peer Workers in Overdose Response Settings. Harm Reduct J [Internet]. 2021 [cited 2021 Feb 17];18(28). Available from: https://harmreductionjournal.biomedcentral.com/articles/10.1186/s12954-020-00449-1

38. Braun V, Clarke V. Using thematic analysis in pyschology. Qual Res Psychol [Internet]. 2006 [cited 2018 Mar 4];3(2). Available from: https://journalsscholarsportal-info.myaccess.library.utoronto.ca/pdf/14780887/v03i0002/77_utaip.xml

39. Nowell LS, Norris JM, White DE, Moules NJ. Thematic Analysis. Int J Qual Methods [Internet]. 2017 Dec 2 [cited 2020 Jan 3];16(1):160940691773384. Available from: http://journals.sagepub.com/doi/10.1177/1609406917733847

40. Thorne S. Interpretive Description: Qualitative Research for Applied Practice (Developing Qualitative Inquiry) 2nd edition. Routledge; 2016. 336 p.

41. UNICEF. Module 1: What are the Social Ecological Model (SEM) and Communication for Development (C4D)? [Internet]. 2016. Available from: www.unicef.org/cbsc/files/Module_1_SEM-C4D.docx

42. Tobergte DR, Curtis S. The ottawa charter for health promotion. J Chem Inf Model. 2013;53(9):1689-99.

43. Butterfoss FD, Kegler MC, Francisco VT. Mobilizing Organizations for Health Promotion: Theories of Organizational Change. In: Health behavior and health education: theory, research, and practice. 4th ed. 2008. p. 335-61.

44. May CR, Mair F, Finch T, MacFarlane A, Dowrick C, Treweek S, et al. Development of a theory of implementation and integration: Normalization Process Theory. Implement Sci [Internet]. 2009 Dec 21 [cited 2021 Feb 22];4(1):29. Available from:

http://implementationscience.biomedcentral.com/articles/10.1186/1748-5908-4-29

45. McEvoy R, Ballini L, Maltoni S, O’Donnell CA, Mair FS, MacFarlane A. A qualitative systematic review of studies using the normalization process theory to research implementation processes [Internet]. Vol. 9, Implementation Science. BioMed Central; 2014 [cited 2021 Feb 22]. p. 2. Available from: http://implementationscience.biomedcentral.com/articles/10.1186/1748-5908-9-2

46. Government of Canada. Canadian Community Health Survey - Canada.ca [Internet]. [cited 2020 Jan 9]. Available from: https://www.canada.ca/en/healthcanada/services/food-nutrition/food-nutrition-surveillance/health-nutrition-surveys/canadian-community-health-survey-cchs.html

47. Ware JE, Kosinski M, Keller SD. A 12-Item Short-Form Health Survey: Construction of Scales and Preliminary Tests of Reliability and Validity. Med Care. 1996;34(3):220-33.

48. The Centre for Victims of Torture. Professional Quality of Life [Internet]. 2012 [cited 2020 Jan 9]. Available from: https://www.proqol.org/

49. Spector P. Job Satisfaction Survey [Internet]. 1994 [cited 2020 Jan 9]. Available from: http://shell.cas.usf.edu/ pspector/scales/jsspag.html

50. Peer-2-Peer Project. \#PeerLife [Internet]. Canada; 2020. Available from: https://www.youtube.com/watch?v=XcMBpGJqwGU\&t=21s

51. BC Centre for Disease Control. Peer Payment Standards for Short-term Engagements [Internet]. Vancouver; 2018 [cited 2020 Nov 16]. Available from: http://www.bccdc.ca/resource-gallery/Documents/Educational Materials/Epid/Other/peer_payment-guide_2018.pdf 
52. Peer-2-Peer Project. Best Practice Manual for Supporting Peer Workers in Overdose Response Settings [Internet]. 2020 [cited 2021 Feb 23 ]. Available from: https://towardtheheart.com/assets/uploads/1610410618yGfdr6sQs8m8KTWoKfaCUWX9llgwxBYHcMkNIFJ.pdf

53. Shapiro A, Sim D, Wu H, Mogg M, Tobias S, Patel P, et al. Detection of etizolam, flualprazolam and flubromazolam by benzodiazepine-specific lateral flow immunoassay test strips. 2020.

54. Jen St. Denis. Why B.C.'s illicit drugs are increasingly deadly. Citizen [Internet]. 2020 Aug 16 [cited 2021 Jan 27]; Available from: https://www.princegeorgecitizen.com/news/local-news/why-b-c-s-illicit-drugs-are-increasingly-deadly-1.24187413

55. Yvette Brend. New drug mix causing harder-to-revive overdoses, sparking panic in Vancouver | CBC News. CBC News [Internet]. 2019 Apr 12 [cited 2021 Jan 27]; Available from: https://www.cbc.ca/news/canada/british-columbia/new-kind-of-overdose-dtes-benzo-filler-anxiety-depression-drug-makingnarcan-fail-1.5096395

56. Diangelo R. "We Are All for Diversity, but ...": How Faculty Hiring Committees Reproduce Whiteness and Practical Suggestions for How They Can Change. Harv Educ Rev [Internet]. 2017 [cited 2018 Apr 12];87(4). Available from: http://hepgjournals.org.ezproxy.library.ubc.ca/doi/pdf/10.17763/1943-504587.4.557

57. Lippert-Rasmussen K. Intentions and Discrimination in Hiring. J Moral Philos [Internet]. 2012 Jan 1 [cited 2018 Apr 12];9(1):55-74. Available from: http://booksandjournals.brillonline.com/content/journals/10.1163/174552411x612065

58. Tetrick LE, Winslow CJ. Workplace Stress Management Interventions and Health Promotion. Annu Rev Organ Psychol Organ Behav [Internet]. 2015 Apr 10 [cited 2021 Feb 10];2(1):583-603. Available from: http://www.annualreviews.org/doi/10.1146/annurev-orgpsych-032414-111341

59. Cobb S. Social Support as a Moderator of Life Stress. Psychosom Med. 1976;38(5).

60. House JS. Work stress and social support [Internet]. Addison-Wesley Pub. Co.; 1981 [cited 2021 Feb 10]. Available from: https://www.worldcat.org/title/work-stress-and-social-support/oclc/654126161

61. Dennis C-L. Peer support within a health care context. Int J Nurs Stud. 2003;40.

62. Livingston JD, Milne T, Fang ML, Amari E. The effectiveness of interventions for reducing stigma related to substance use disorders: a systematic review. Addiction [Internet]. 2012 Jan 1 [cited 2021 Feb 10];107(1):39-50. Available from: http://doi.wiley.com/10.1111/j.1360-0443.2011.03601.x

63. Wallace B, Pagan F, Pauly B (Bernie). The implementation of overdose prevention sites as a novel and nimble response during an illegal drug overdose public health emergency. Int J Drug Policy [Internet]. 2019 Apr 1 [cited 2020 Sep 11];66:64-72. Available from:

https://pubmed.ncbi.nlm.nih.gov/30708237/

\section{Figures}

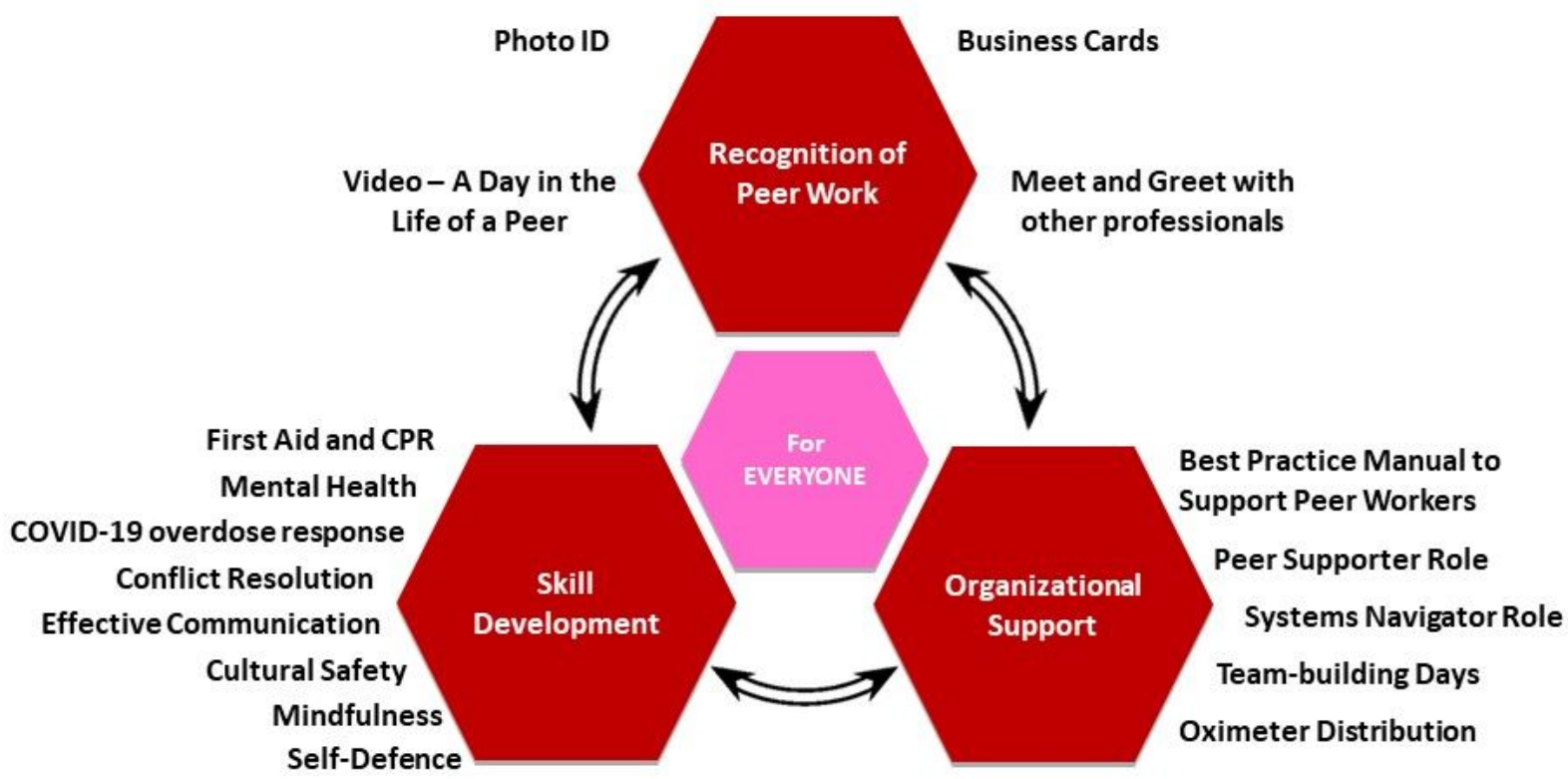


Figure 1

Components of the ROSE Model and the strategies within each component

\section{Supplementary Files}

This is a list of supplementary files associated with this preprint. Click to download.

- ROSEPaperCOREQChecklist.pdf 